\title{
Strengthened tuberculosis control programme and trend of multidrug resistant tuberculosis rate in Osaka City, Japan
}

Akira Shimouchi, ${ }^{a}$ Akihiro Ohkado, ${ }^{a}$ Kenji Matsumoto, b Jun Komukai, ${ }^{b}$ Hideki Yoshidab and Nobukatsu Ishikawa ${ }^{a}$ Correspondence to Akira Shimouchi (e-mail: shimouchi@jata.or.jp).

Osaka City has the highest tuberculosis (TB) notification rates in Japan. In the period 1999-2003, the TB control programme was strengthened, and the Stop TB Strategy was implemented to reduce the number of notified cases. The objective of this study was to assess the effect of these control activities in Osaka City, including the implementation of directly observed treatment (DOT), by analysing TB surveillance and routinely collected data. We reviewed the surveillance data of all sputum smear-positive pulmonary tuberculosis (PTB) cases registered in the Osaka City Public Health Office from 2001 to 2008 and data collected from the routine TB programme. The DOT implementation rate increased from 0\% in 2001 to $68 \%$ in 2008 for smear-positive PTB cases of the general public and to $61 \%$ for all PTB cases of the homeless. The proportion of smear-positive PTB cases that had treatment failure and default combined, declined from $8.0 \%$ (52 of 650 ) in 2001 to $3.6 \%$ (20 of 548 ) in 2006 . The proportion of cases among the homeless with previous treatment declined from $28 \%$ in 2001 to $15 \%$ in 2008 . The proportion of cases with multidrug resistant-TB (MDR-TB) among those without previous treatment declined from $1.7 \%$ in 2001 to $0.9 \%$ in 2008 . It is logical that reduction in the failure and default rate would lead to the reduction of cases with previous treatment and TB transmission, including resistant TB, therefore to the reduction of MDR-TB rates.

O saka City is the third largest city (population of 2.6 million in 2005) and has the highest tuberculosis (TB) notification rate in Japan. The TB notification rate in Osaka City decreased during the 1970s, but this decline slowed substantially and has stagnated since $1983 .{ }^{1}$ During this period, the notification rate of Osaka City was two to three times higher than the national rate. This stagnation of decline occurred in only a few wards (districts) where unemployment rates were high among the middle-aged. In 1998, a cohort analysis in an inner city ward, Naniwa, showed that $30 \%$ of TB cases defaulted from their treatment. ${ }^{2}$ In 1997 , $42.9 \%$ of homeless TB cases registered in Osaka City had been previously treated. This declined to $27.4 \%$ in $2001 .^{3}$

During the period 1999-2003, to strengthen the TB control programme, the Osaka City Government implemented the following:

- Osaka City Public Health Office (OCPHO) was newly established in 1999, and the 24 ward- level TB Diagnostic Committees (TBDC) were integrated into one as part of OCPHO;

- OCPHO became responsible for the integrated TB programme for homeless cases in the city;

- from the year 2000 , public health nurses were allowed to visit TB cases outside of Osaka City routinely (previously they were not allowed due to budget constraints);

- the STOP TB Strategy, 10-Year Plan of Tuberculosis Control in Osaka City 2001-2010 4 was launched in 2001; and

- eight public health nurse positions were created at OCPHO for directly observed treatment (DOT) and programme implementation for the homeless in 2003.

The target of the "STOP TB Strategy" was to halve the TB notification rate in Osaka City to 50 per 100000 population by $2010 .{ }^{4}$ The main strategies to do this

\footnotetext{
The Research Institute of Tuberculois, Japan Anti-Tuberculosis Association (RIT JATA), Tokyo Japan.

Osaka City Public Health Office, Osaka, Japan.

Submitted: 05 November 2012; Published: 26 February 2013

doi: 10.5365/wpsar.2012.3.4.015
} 
included the promotion of the standard treatment regimen containing pyrazinamide (PZA), treatment support by using DOT in hospitals and the community and monitoring and evaluating case management. The treatment regimen was to be approved by the TBDC as required by law, ${ }^{5}$ and any changes to the regimen were to be advised to clinicians by the TBDC as necessary.

In Japan, smear-positive pulmonary TB (PTB) cases are legally required to be isolated in hospitals until their smears or cultures are negative. ${ }^{5}$ During this hospitalization, most cases are treated with DOT by nurses. After discharge from hospital, treatment support can be provided by DOT nurses through weekly visits to the cases' houses or cases visiting a pharmacy weekly. For all PTB cases of the homeless, daily DOT is provided at the DOT clinic of a semi-governmental hospital located in a poor area of $0.6 \mathrm{~km}^{2}$ where 21000 of the 30000 total population are daily-paid laborers and homeless people living in small hostels, shelters, parks or on roads. ${ }^{6}$

For monitoring and evaluation of case management, cohort analysis meetings are held at all 24 ward public health offices and OCPHO. Information such as treatment regimen, drug susceptibility testing (DST) results and type of case support were obtained. One case was to be discussed twice during the treatment so that any necessary change of regimen or case support could be undertaken.

Notification of TB cases is mandatory in Japan, ${ }^{5}$ with every case of diagnosed TB reported by a clinician to the local public health office. Individual data are recorded on a case management card by public health nurses of the 24 ward public health offices and OCPHO. Data included on the card are name, date of birth, sex, occupation, co-morbidities, diagnosis, results of bacteriological examination, chest X-ray findings, regimen and treatment outcome. This data are further entered into the computerized surveillance system. DST results were not systematically collected before 2001.

The objective of this study was to assess the effect of the TB control programme, including DOT, in Osaka City by assessing TB surveillance and routinely collected data from the programme, including the rate of multidrug resistant-TB (MDR-TB) between 2001 and 2008.

\section{METHODS}

This is a descriptive and observational study using TB surveillance and routinely collected data by the TB control programme. All smear-positive PTB cases registered in Osaka City from 2001 to 2008 were included for DST results.

The indicators used included the proportion of all PTB cases using PZA, DOT implementation rate for all smear-positive PTB cases of the general public and for all PTB cases of the homeless, previous treatment case rate of all PTB cases for the general public and the homeless and treatment outcome of smear-positive PTB cases. MDR-TB cases and remainders were assessed by previous treatment status and other factors for smearpositive PTB cases.

The homeless are defined in this study as people who live in parks, on river banks, roads, stations or other facilities without permission. ${ }^{7}$ The treatment outcomes are defined as follows: failure - positive culture result five to 12 months after the start of treatment; default - treatment is interrupted for more than two months in total or treatment ended in less than standard duration; ${ }^{8}$ and case previously treated - a case treated with anti-TB medicine for at least one month and at least two months before current treatment. MDR-TB was defined as a case resistant to both Isoniazid (INH) and rifampicin (RFP).

DSTs are conducted according to the guidelines in Japan ${ }^{9}$ in three public hospitals and four private hospitals in Osaka Prefecture and at the national TB reference hospital. Quality assurance programmes of DST have showed reliable results for RFP and INH. ${ }^{10}$

Aggregated data and the electronic database of registered cases without name and date of birth were used and analysed. The $\chi^{2}$ test was used for trend analysis of categorical data using Epi Info, and $P$-values were used for judging statistical significance.

A multivariate analysis was constructed using logistic regression models in SPSS to compare MDRTB cases with the remainder. Data were only available at the individual level for age, sex, previous treatment and residence; therefore, these were used in the model. Only cases with all these fields completed were included. The step-wise maximum likelihood estimation 
Figure 1. Notification rate of all TB and smear-positive pulmonary TB, Osaka City, Japan, 2001 to 2008

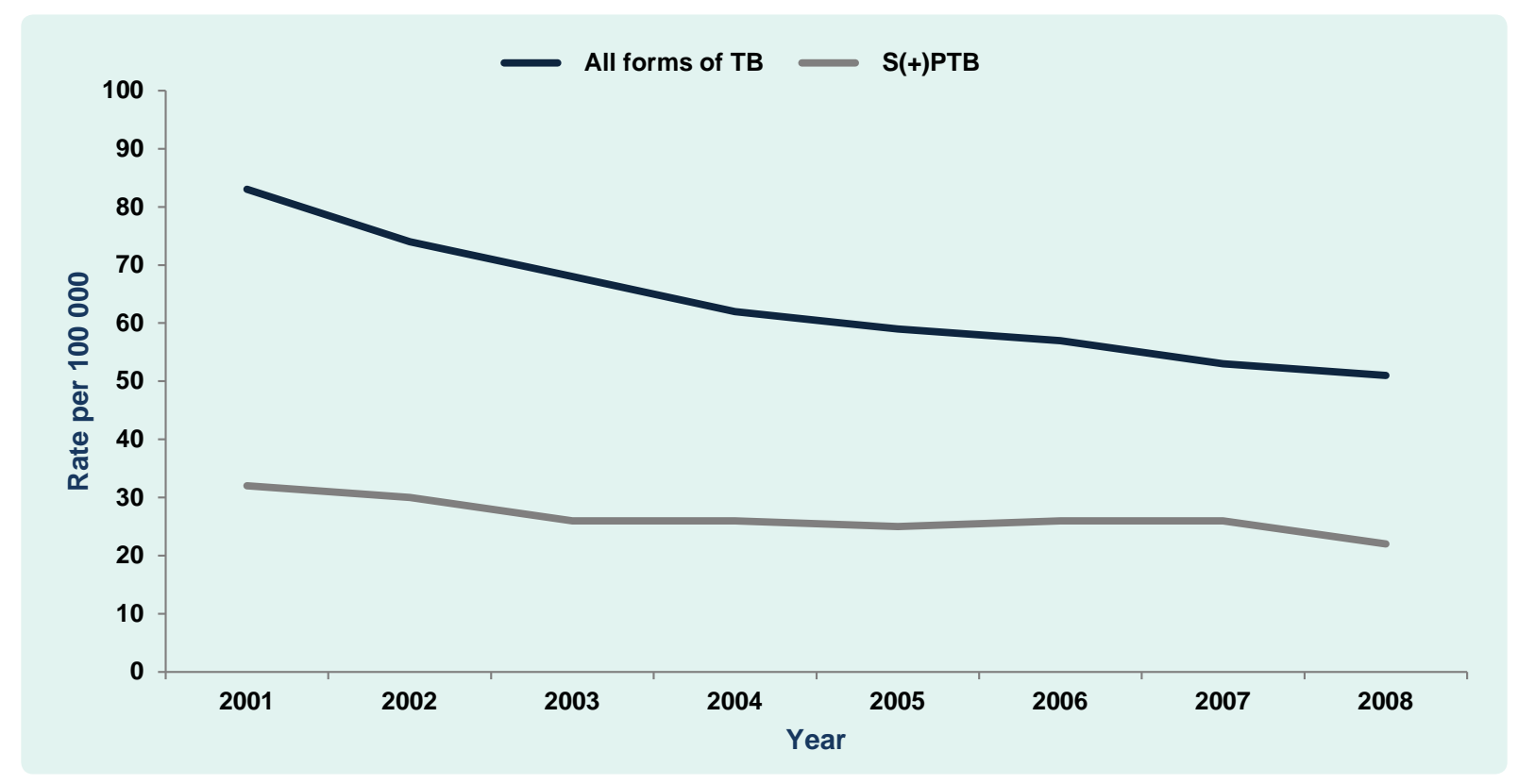

TB - tuberculosis; S(+)PTB - smear-positive pulmonary tuberculosis

with a significance level of $<0.2$ was used to select the variables for the logistic regression models.

As the data used in this study were collected through routine surveillance mechanisms by public health authorities and did not contain any identifying data, the office for ethical issues in Osaka City Government deemed it not necessary to obtain the ethical clearance.

\section{RESULTS}

There were 5589 new smear-positive PTB cases registered between 2001 and 2008; the number declined from 821 in 2001 to 569 in 2008. DST results were available for 5030 (90\%) of these cases. The notification rate of all TB and smear-positive TB cases declined from 2001 to 2008 (Figure 1).

From 2001 to 2008, the proportion of all PTB cases treated with a regimen containing PZA was maintained at more than $70 \%$ each year. DOT implementation rates for smear-positive PTB cases of the general public increased from $0 \%$ in 2001 to $68 \%$ in 2008 (321 of 473) and increased from $0 \%$ to $61 \%$ (114 of 187) for all PTB cases of the homeless during the same period (Figure 2).

The proportion of all PTB cases with previous treatment in the general public was stable over the study period and was $13.5 \%$ overall (1931 of 14 292). For homeless cases, the proportion with previous treatment declined from $27.4 \%$ (97 of 354 ) in 2001 to $15.1 \%$ (22 of 146) in 2008 (Figure 3 ). The proportion of all smear-positive PTB cases that had treatment failure and default combined declined from $8.0 \%$ (52 of 650 ) in 2001 to $3.6 \%$ (20 of 548 ) in 2006.

For all smear-positive PTB cases, the proportion of cases with resistance to INH significantly declined from $9.3 \%$ (63 of 681 ) in 2001 to $5.3 \%$ (27 of 505 ) in $2008(P=0.016)$. Similarly, the proportion of cases with resistance to RFP significantly declined from 3.4\% (23 of 681) in 2001 to $2.2 \%$ (11 of 505 ) in 2008 $(P=0.015)$. The proportion of cases with MDR-TB also significantly declined from $2.6 \%$ (18 of 681) in 2001 to $1.8 \%$ (nine of 505$)$ in $2008(P=0.017)$ (Table 1 ).

The proportion of cases without previous TB treatment with resistance to $\mathrm{INH}$ declined from $6.4 \%$ (37 of 579 ) in 2001 to $4.0 \%$ (18 of 446 ) in 2008 , although this was not statistically significant $(P=0.212)$. There was also a decline from $2.4 \%$ (14 of 579 ) in 2001 to $1.1 \%$ (5 of 446) in 2008 for resistance to RFP in these cases, and this was statistically significant $(p$-value $=0.002)$. MDR-TB also significantly declined from $1.7 \%$ (10 of 579 ) in 2001 to $0.9 \%$ (4 of 446 ) in $2008(P=0.0173)$ for those cases without previous TB treatment (Table 1). 
Figure 2. Proportion of TB cases using PZA and DOT and the proportion of homeless cases using DOT, Osaka City, Japan, 2001 to 2008

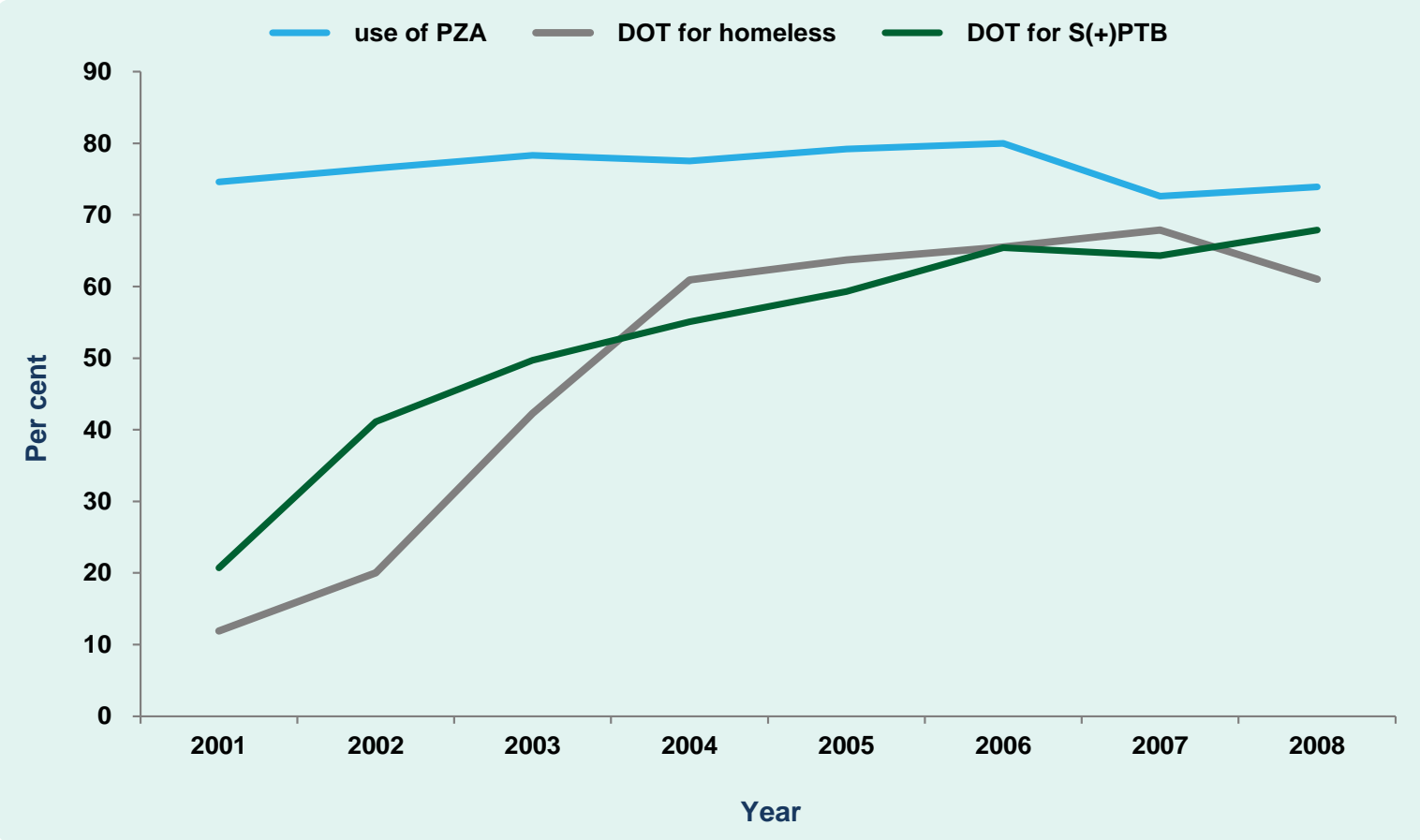

TB - tuberculosis; PZA - pyrazinamide; DOT - directly observed treatment; S(+)PTB - smear-positive pulmonary tuberculosis

Figure 3. Proportion of TB cases with previous treatment in the general public and for the homeless, Osaka City, Japan, 2001 to 2008

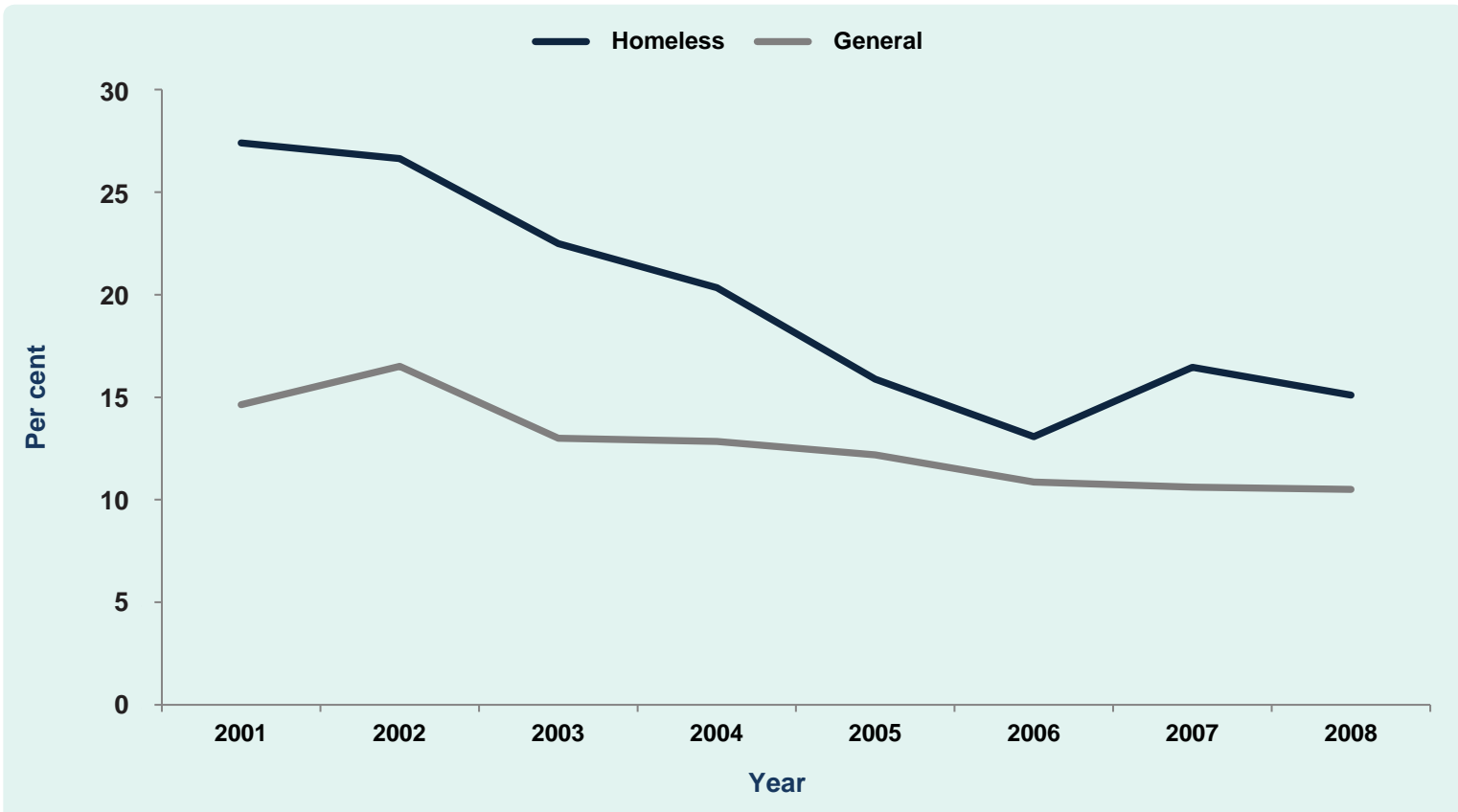

For those cases that had previous treatment, the proportion of resistance to INH, or RFP or MDR-TB did not show any significant reduction from 2001 to 2008 (Table 1).
When comparing MDR-TB cases $(n=81)$ with the remainder of cases $(n=4609)$ in the multivariate model, having MDR-TB was significantly associated with having had previous treatment (crude OR: 7.89; 
Table 1. Number and proportion of smear-positive pulmonary TB cases with drug resistance to isoniazid and/or rifampicin by treatment status, Osaka City, Japan, 2001 to 2008

\begin{tabular}{|c|c|c|c|c|c|c|c|c|c|c|c|c|c|c|c|c|c|c|c|c|c|}
\hline \multirow{3}{*}{ Year } & \multicolumn{7}{|c|}{ Combined } & \multicolumn{7}{|c|}{ Without previous treatment } & \multicolumn{7}{|c|}{ With previous treatment } \\
\hline & \multirow{2}{*}{$\begin{array}{c}\text { All } \\
\text { cases* }^{*}\end{array}$} & \multicolumn{2}{|c|}{ any $\mathrm{H}$} & \multicolumn{2}{|c|}{ any $R$} & \multicolumn{2}{|c|}{ any $\mathrm{RH}$} & \multirow{2}{*}{$\begin{array}{c}\text { All } \\
\text { cases* }^{*}\end{array}$} & \multicolumn{2}{|c|}{ any $H$} & \multicolumn{2}{|c|}{ any $R$} & \multicolumn{2}{|c|}{ any $\mathrm{RH}$} & \multirow{2}{*}{$\begin{array}{c}\text { All } \\
\text { cases* }^{*}\end{array}$} & \multicolumn{2}{|c|}{ any $H$} & \multicolumn{2}{|c|}{ any $R$} & \multicolumn{2}{|c|}{ any $\mathrm{RH}$} \\
\hline & & $n$ & $\%$ & $n$ & $\%$ & $n$ & $\%$ & & $n$ & $\%$ & $n$ & $\%$ & $n$ & $\%$ & & $n$ & $\%$ & $n$ & $\%$ & $n$ & $\%$ \\
\hline 2001 & 681 & 63 & 9.3 & 23 & 3.4 & 18 & 2.6 & 579 & 37 & 6.4 & 14 & 2.4 & 10 & 1.7 & 102 & 26 & 25.5 & 9 & 8.8 & 8 & 7.8 \\
\hline 2002 & 664 & 50 & 7.5 & 27 & 4.1 & 17 & 2.6 & 546 & 29 & 5.3 & 15 & 2.7 & 6 & 1.1 & 118 & 21 & 17.8 & 12 & 10.2 & 11 & 9.3 \\
\hline 2003 & 651 & 37 & 5.7 & 19 & 2.9 & 16 & 2.5 & 568 & 33 & 5.8 & 16 & 2.8 & 13 & 2.3 & 83 & 4 & 4.8 & 3 & 3.6 & 3 & 3.6 \\
\hline 2004 & 633 & 36 & 5.7 & 11 & 1.7 & 7 & 1.1 & 541 & 32 & 5.9 & 7 & 1.3 & 4 & 0.7 & 92 & 4 & 4.3 & 4 & 4.3 & 3 & 3.3 \\
\hline 2005 & 632 & 26 & 4.1 & 10 & 1.6 & 5 & 0.8 & 555 & 19 & 3.4 & 4 & 0.7 & 2 & 0.4 & 77 & 7 & 9.1 & 6 & 7.8 & 3 & 3.9 \\
\hline 2006 & 644 & 38 & 5.9 & 9 & 1.4 & 4 & 0.6 & 571 & 28 & 4.9 & 6 & 1.1 & 2 & 0.4 & 73 & 10 & 13.7 & 3 & 4.1 & 2 & 2.7 \\
\hline 2007 & 620 & 45 & 7.3 & 18 & 2.9 & 12 & 1.9 & 548 & 35 & 6.4 & 7 & 1.3 & 5 & 0.9 & 72 & 10 & 13.9 & 11 & 15.3 & 7 & 9.7 \\
\hline 2008 & 505 & 27 & 5.3 & 11 & 2.2 & 9 & 1.8 & 446 & 18 & 4.0 & 5 & 1.1 & 4 & 0.9 & 59 & 9 & 15.3 & 6 & 10.2 & 5 & 8.5 \\
\hline
\end{tabular}

$\mathrm{H}$ - isoniazid; $\mathrm{R}$ - rifampicin

*All smear positive pulmonary tuberculosis cases that drug susceptibility result is available.

Table 2. Comparison between multidrug resistant TB cases and all other positive pulmonary TB cases, Osaka City, Japan, 2001 to 2008

\begin{tabular}{|c|c|c|c|c|c|c|c|c|c|}
\hline \multicolumn{2}{|c|}{ Multidrug resistance } & \multirow{2}{*}{$\begin{array}{c}\text { Yes } \\
16\end{array}$} & \multirow{2}{*}{$\begin{array}{c}\text { No } \\
3562\end{array}$} & \multirow{2}{*}{$\begin{array}{c}\text { Crude OR } \\
1.00\end{array}$} & \multirow{3}{*}{$\begin{array}{c}95 \% \mathrm{Cl} \\
0.70-2.21\end{array}$} & \multirow{3}{*}{$\begin{array}{c}P \text { value } \\
0.462\end{array}$} & \multirow{3}{*}{$\begin{array}{c}\text { Adjusted OR* } \\
-\end{array}$} & \multirow{3}{*}{$\begin{array}{c}95 \% \mathrm{Cl} \\
-\end{array}$} & \multirow{3}{*}{$\begin{array}{c}P \text { value } \\
-\end{array}$} \\
\hline Age & Less than 40 & & & & & & & & \\
\hline & 40 and above & 16 & 1047 & 1.24 & & & & & \\
\hline \multirow[t]{2}{*}{ Sex } & Female & 15 & 856 & 1.00 & \multirow{2}{*}{$0.38-1.28$} & \multirow{2}{*}{0.249} & \multirow{2}{*}{ - } & \multirow{2}{*}{-} & \multirow{2}{*}{-} \\
\hline & Male & 66 & 3753 & 0.70 & & & & & \\
\hline \multirow[t]{2}{*}{ Treatment } & $\begin{array}{l}\text { Without previous } \\
\text { treatment }\end{array}$ & 40 & 4141 & 1.00 & \multirow[b]{2}{*}{$4.96-11.57$} & \multirow[b]{2}{*}{$<0.001$} & \multirow[b]{2}{*}{7.57} & \multirow[b]{2}{*}{$4.78-11.98$} & \multirow[b]{2}{*}{$<0.001$} \\
\hline & $\begin{array}{l}\text { With previous } \\
\text { treatment }\end{array}$ & 41 & 509 & 7.89 & & & & & \\
\hline \multirow[t]{2}{*}{ Residence } & $\begin{array}{l}\text { Any ward except } \\
\text { Nishinari }\end{array}$ & 46 & 3307 & 1.00 & \multirow{2}{*}{$1.07-2.81$} & \multirow{2}{*}{0.0027} & \multirow{2}{*}{1.58} & \multirow{2}{*}{$0.99-2.52$} & \multirow{2}{*}{0.053} \\
\hline & $\begin{array}{l}\text { Homeless, } \\
\text { Nishinari Ward }\end{array}$ & 35 & 1348 & 1.73 & & & & & \\
\hline
\end{tabular}

Crude OR - crude odds ratio; Adjusted OR - adjusted odds ratio; $\mathrm{Cl}$ - confidence interval.

* Logistic regression adjusted for the four variables in this table.

95\% Cl: 4.96-11.57; $P<0.001$, adjusted OR: 7.57; 95\% Cl: 4.78-11.98; $P<0.001$ ) and being a resident of Nishinari ward or homeless (crude OR: $1.73 ; 95 \% \mathrm{Cl}$ : 1.07-2.81, adjusted OR: $1.58 ; 95 \% \mathrm{Cl}: 0.99-2.52$; $P=0.053$ ). Having MDR-TB was not associated with sex or age group (Table 2).

\section{DISCUSSION}

Since the strengthening of the TB control programme in Osaka City and the implementation of the STOP TB
Strategy, all TB indicators have improved. The goal of the Stop TB Strategy to reduce the TB notification rate to 50 per 100000 by $2010^{4}$ was almost reached in 2008 with a notification rate of 51 per 100000 . Improvements in TB indicators included increases in the proportion of cases using PZA in their treatment regimen and the implementation rate of DOT, reductions in the proportion of cases with treatment failure and default, and reductions in the proportion of previous treatment of the homeless and the proportion of cases with drug resistance, including 
MDR-TB. The proportion of homeless cases with previous treatment declined before DOT started in 1997 because PZA was introduced in 1996 in Japan, and PZA usage rates had already reached $56 \%$ before DOT started. As all PTB homeless cases including resistant TB were targeted for daily DOT, the treatment outcome of resistant TB cases should also be improved. Thus it would be logical that the reduction of the failure and default rate would lead to the reduction of the previous treatment rate and to the reduction of TB transmission, including resistant $\mathrm{TB}$, in the community.

The homeless are a high-risk group for TB infection in Osaka City; this has also been reported in the United States of America where drug-resistant TB is also significantly higher in homeless individuals, ${ }^{11}$ and in London where homelessness was associated with the risk of multidrug resistance, poor adherence to treatment and loss to follow-up. ${ }^{12}$ DOT for homeless cases has been introduced in urban areas of Japan such as Tokyo, Yokohama, Kawasaki and Nagoya since 1997 and has resulted in improved treatment success rates. ${ }^{13,14}$ In Shinjuku, Tokyo, after the introduction of DOT for the homeless and foreigners from 2000 to 2006 , the re-treatment rates decreased from $19.4 \%$ to $10.0 \%(P<0.001)$ and MDR-TB rates from $1.6 \%$ to $0.2 \%(P=0.042){ }^{15}$ The same findings were observed with this study, suggesting that strengthening control activities for this high-risk group, including the use of DOT, can be effective.

The main limitation of this report is that only smear-positive PTB cases were studied as these are the only cases for which drug resistance is recorded. Based on surveillance data for $2008,{ }^{16}$ our sample potentially missed an additional $40 \%$ of cases that were bacteriologically confirmed but smear-negative. As a result of this study, the drug resistance of all bacteriologically positive TB cases are now being recorded. Another limitation for our multivariate analysis is that the individual case data did not contain occupation, education, use of PZA, type of DOT and other factors, and therefore these could not be included in the model. Lastly, as the data are from routine systems, the entering rate might have varied among different ward public health offices.

Despite these limitations, this analysis of TB surveillance and routinely collected data from the programme showed a statistically significant reduction in the proportion of TB cases with drug resistance from 2001 to 2008. This most likely resulted from the improvements in treatment outcome which were made possible by strengthening TB control activities, including DOT implementation for homeless cases, in Osaka city.

\section{Conflicts of interest}

None declared.

Funding

The study was financially supported by the Health Research Fund of Ministry of Health, Labour \& Welfare, Japan.

\section{Acknowledgements}

The authors express their gratitude for all the staff of the Infectious Disease Division of Osaka City Public Health Center for their dedicated work on the data collection and analysis.

\section{References:}

1. Takatorige $\mathrm{T}$ et al. [Factors related to the slowdown in the reduction of the tuberculosis incidence rate in Osaka City-structure of the high incidence rate of tuberculosis in Osaka City analyzed by administrative-ward group, five-year period and age group] [in Japanese]. Kekkaku, 2000, 75:533-544. pmid:11068370

2. [Standard treatment of tuberculosis] [in Japanese]. Tokyo, Ministry of Health, Labour and Welfare, amended in 1996.

3. [Tuberculosis in Osaka City 2009, tuberculosis statistics of 2008] [in Japanese]. Osaka, City Public Health Office, 2009.

4. [10-Year Plan of Tuberculosis Control in Osaka City 2001-2010] [in Japanese]. Osaka, STOP TB Strategy and City Public Health Office, 2001.

5. [Law for prevention and medical services for infectious diseases] [in Japanese]. Tokyo, Ministry of Health, Labour and Welfare, 2011 (http://law.e-gov.go.jp/htmldata/H10/H1OHO114.html, accessed 30 October 2012).

6. [Report on programme: Measurement for homeless and Airin Area] [in Japanese]. Osaka, Health and Welfare Bureau and Osaka City Government, 2007 (http://www.city.osaka.Ig.jp/fukushi/ page/0000008085.html, accessed 13 October 2012.

7. [Special law for support of self-reliance of the homeless, enacted on 7 August 2002 - Law No.105, amended on 26 June 2012 Law No. 46] [in Japanese]. Tokyo, Ministry of Health, Labour and Welfare, 2012

8. Tuberculosis Surveillance Center. [RIT, JATA Tuberculosis annual report 2009 - Series 10. Treatment outcome and TB deaths] [in Japanese]. Kekkaku, 2012, 87:415-419. pmid:22696774

9. [Guidelines for laboratory for Mycobacterium tuberculosis] [in Japanese]. Tokyo, Laboratory for Mycobacterium Committee, Japan Tuberculosis Society edt, Japan Anti-Tuberculosis Association, 2007. 
10. Shimouchi A, Tuberculosis Surveillance Research Unit. Trend of multidrug resistance tuberculosis rate in Osaka City, Progress Report 2006. The Netherlands City, KNCV, 2006.

11. Barclay DM 3rd, Richardson JP, Fredman L. Tuberculosis in the homeless. Archives of Family Medicine, 1995, 4:541-546. doi:10.1001/archfami.4.6.541 pmid:7773431

12. Story A et al.; London Tuberculosis Nurses Network. Tuberculosis in London: the importance of homelessness, problem drug use and prison. Thorax, 2007, 62:667-671. doi:10.1136/ thx.2006.065409 pmid:17289861
13. Toyota E. [DOTS in Japan-Tokyo area] [in Japanese]. Kekkaku, 2001, 76:749-753. pmid:11806131

14. Tada $Y$ et al. [Tuberculosis control in Kawasaki City-promoting the DOT program] [in Japanese]. Kekkaku, 2004, 79:17-24. pmid:14969084

15. Kaguraoka S et al. [Tuberculosis control in Shinjuku Ward, Tokyopromoting the DOTS program and its outcome] [in Japanese]. Kekkaku, 2008, 83:611-620. pmid:18979995

16. [Official data submitted to the evaluation meeting of Osaka City Public Health Office in December 2012] [in Japanese]. 